\title{
A Prospective Study of Thyroid Dysfunction in Patients with Type2 Diabetes Mellitus in A Tertiary Care Hospital (Faamch, Barpeta, Assam, India)
}

\author{
Dr. Anuradha Deuri ${ }^{1}$, Dr. J. Thakuria ${ }^{2}$, Dr. D. Kalita ${ }^{3}$ \\ ${ }^{I}$ associate Professor Of Medicine, Faa Medical College Hospital, Barpeta, Assam. \\ ${ }^{2}$ professor \&Head, Department Of Medicine, Faa Medical College Hospital, Barpeta, Assam. \\ 3 assistant Professor Of Paediatrics, Gauhati Medical College Hospital, Guwahati, Assam.
}

\begin{abstract}
:
Background: Diabetes mellitus is one of the most common metabolic disorder with its increasing incidence world wide. Thyroid dysfunction is another common endocrine disorder frequently encountered in clinical practice(1). The association between diabetes mellitus and thyroid disorder is widely known. Under this backdrop, the present study was undertaken with the following aims and objectives. 1) To know the prevalence of thyroid dysfunction in Type 2 diabetes mellitus. 2)To know the spectrum of thyroid dysfunction in Type 2 diabetes mellitus.

Methods: This hospital based prospective study was conducted in the department of Medicine, FAA Medical College \& hospital, Barpeta, Assam, India over a period of one year from May 2015 to April 2016. A total number of 120 patients with Type 2 diabetes mellitus were included in the study. Data collection was done by history taking, clinical examination and essential laboratory tests.

Results: Out of 120 patients , 27 (22.5\%) had thyroid dysfunction and remaining 93 patients had normal thyroid function. In this study, out of 27 thyroid dysfunction patients, 8 (6.6\%) had clinical hypothyroidism , 17 (14.1\%) had subclinical hypothyroidism and 2 (1.6\%) diabetics had hyperthyroidism. Thyroid dysfunction was seen more in female $18(15 \%)$ cases than in male $9(7.5 \%)$ cases. Thyroid dysfunction was more common in diabetic patients above 60 years of age. Further, diabetics with microvascular complications had more thyroid dysfunctions.

Conclusion: In conclusion, the present study showed a high prevalence of thyroid dysfunction (22.5\%) among the diabetic patients studied. Subclinical hypothyroidism was more common

than clinical hypothyroidism. It is known that undiagnosed thyroid dysfunction in diabetics increases the cardiovascular risk in these patients. Hence, screening for thyroid dysfunction among diabetic patients should be routinely performed to reduce the morbidity and mortality.
\end{abstract}

Keywords: Type 2 Diabetes mellitus, hypothyroidism, subclinical hypothyroidism, hyperthyroidism, nephropathy, neuropathy, retinopathy.

\section{Introduction}

Diabetes mellitus is one of the most common metabolic disorder with its increasing incidence world wide. It has become one of the most leading cause of morbidity and mortality. The metabolic dearrangement in relation to diabetes mellitus in other word causes secondary pathophysiological changes in multiple organ system leads to complications. The common microvascular complications of diabetes mellitus are nephropathy, neuropathy and retinopathy etc. Thyroid dysfunction is another common endocrine disorder frequently encountered in clinical practice(1). The association between diabetes mellitus and thyroid disorder is widely known and the first study was published in 1979 (2). After that, several studies were conducted in different countries to see the prevalence of thyroid dysfunction in diabetic patients. The prevalence of thyroid dysfunction varies widely in community level in different countries, ranging from $6.6 \%$ to $13.4 \%$. (3 ). But, the prevalence of thyroid dysfunction in diabetes mellitus is higher and ranges from 10 to $29 \%(1,3,4)$. This variability of prevalence is usually due to different diagnostic criteria of thyroid disorder, degree of iodine intake in different region, different sensitivities of the TSH assays and the large population diversity (5). It is seen that many diabetic patients may remain asymptomatic especially in subclinical hypothyroidism. Hence, screening of diabetic patients for thyroid disorder is justified for early detection. Many studies were conducted in different countries, but very little data is available in our country especially in the state of Assam. Under this backdrop, the present study was undertaken with the following aims and objectives. 1) To know the prevalence of thyroid dysfunction in Type 2 diabetes mellitus. 2) To know the spectrum of thyroid dysfunction in Type 2 diabetes mellitus. 


\section{Methods}

This hospital based prospective study was conducted in the department of Medicine, FAA Medical College and hospital, Barpeta, Assam, India over a period of one year from May 2015 to April 2016. This Institution is a tertiary health care centre located in rural area in the state of Assam, India and mainly cater to the middle and lower class people of rural Assam. A total number of 120 patients with Type 2 diabetes mellitus that regularly attended the outpatient clinic and also admitted patients who were taking treatment in the hospital regularly were included in the study. Out of 120 patients, 60 male and 60 female patients were selected randomly.

Patients were diagnosed based on the ADA criteria for diabetes.

$>$ Symptoms of diabetes plus random blood glucose concentration of $11.1 \mathrm{mmol} / \mathrm{L}(200 \mathrm{mg} / \mathrm{dl})$.

Fasting plasma glucose of $7.0 \mathrm{mmol} / \mathrm{L}(126 \mathrm{mg} / \mathrm{dl})$.

Or

$>$ Two hour plasma glucose of $11.1 \mathrm{mmol} / \mathrm{L}(200 \mathrm{mg} / \mathrm{dl})$ during an oral glucose tolerance test.

Inclusion criteria:

1) Patient with Type 2 diabetes mellitus with age above 30 years and duration of diabetes longer than 6 months were taken.

2) Type 2 diabetic Patients irrespective of glucose control and treatment (oral drugs/insulin).

Exclusion criteria:

1) Type 1 diabetes mellitus.

2) Patients with gestational diabetes mellitus and steroid induced diabetes.

3) Patients who had proven pre existing thyroid dysfunction.

A written informed consent was taken from all patients and purpose of the study was explained to them. Patient were allowed to discontinue from study at any time.

The study protocol was approved by the Institutional ethics committee of FAA Medical College and hospital, Assam, India.

After taking the history in details , clinical examination was performed with special reference to thyroid gland and other microvascular complications of diabetes mellitus. All clinical findings were noted in the proforma. All patients underwent laboratory evaluation. Diabetes status of the patients were estimated by analyzing FBS, PPBG and RBS. All diabetics underwent target organ evaluation in addition to their routine urine and haematological workup. Patients were also evaluated for thyroid function status by estimation of T3, T4 and TSH level. All haematological and routine urine tests were done in the central laboratory of the Medical College hospital. FNAC and biopsy of thyroid gland was done by our pathologist in the Medical College hospital . Additional laboratory tests were done as per the need of the patient.

\section{Results}

A total number of 120 diabetes mellitus Type 2 patients were included in the study. Out of 120 patients , $27(22.5 \%)$ had thyroid dysfunction and remaining 93 patients had normal thyroid function.

Table- 1 showing prevalence of thyroid dysfunction in Type 2 Diabetes mellitus cases under study $(\mathbf{n = 1 2 0})$

\begin{tabular}{|l|l|}
\hline Thyroid dysfunction & No. of patients. \\
\hline Hypothyroidism & $8(6.6 \%)$ \\
\hline Subclinical hypothyroidism & $17(14.1 \%)$ \\
\hline Hyperthyroidism & $2(1.6 \%)$ \\
\hline Normal & $93(77.5 \%)$ \\
\hline Total & 120 \\
\hline
\end{tabular}

In this study, out of 27 thyroid dysfunction patients, 8 (6.6\%) had clinical hypothyroidism , $17(14.1 \%)$ had subclinical hypothyroidism and $2(1.6 \%)$ diabetics had hyperthyroidism. 


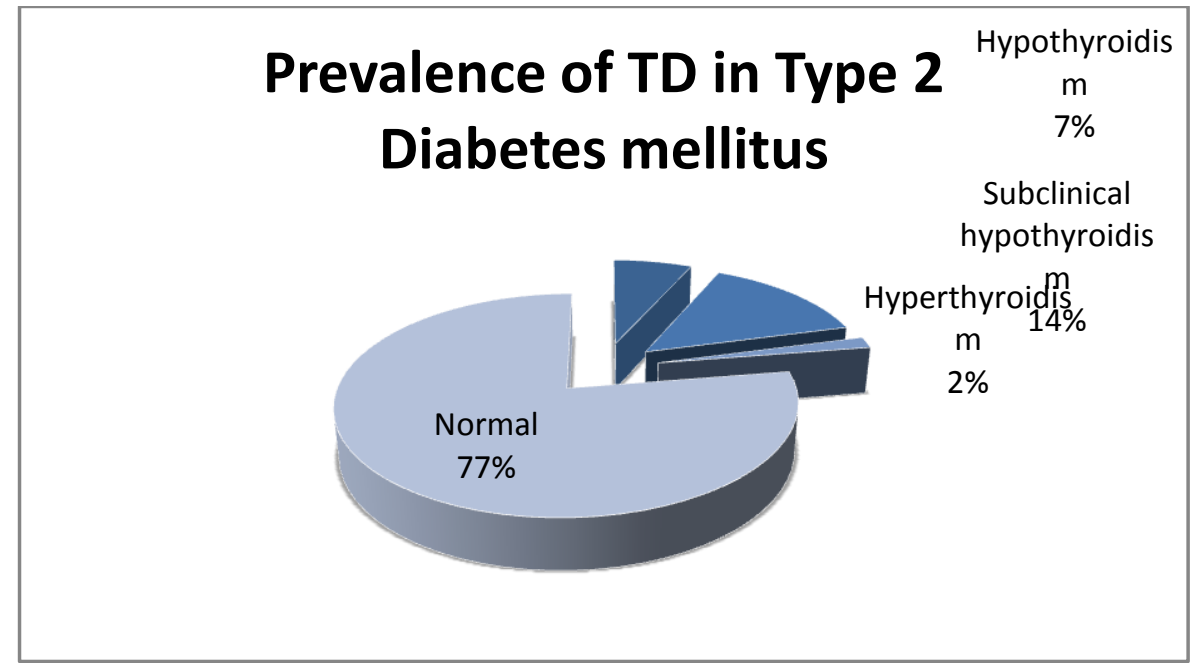

Table 2 shows sex wise distribution of thyroid dysfunction in Type 2 Diabetes mellitus cases $(\mathrm{n}=120)$

\begin{tabular}{|l|l|l|l|l|l|}
\hline Sex & Hypothyroidism & $\begin{array}{l}\text { Subclinical } \\
\text { hypothyroidism }\end{array}$ & Hyperthyroidism & Normal & Total \\
\hline Male & 3 & 5 & 1 & 51 & 60 \\
\hline Female & 5 & 12 & 1 & 42 & 60 \\
\hline
\end{tabular}

In the present study, we randomly selected 60 male and 60 female diabetic patients. We found thyroid dysfunction more in female $18(15 \%)$ cases than in male $9(7.5 \%)$ cases. Out of 18 female patients, 5(4.1\%) had clinical hypothyroidism and $12(10 \%)$ patients had subclinical hypothyroidism . Hyperthyroidism was seen in only $1(0.8 \%)$ patient. Among male diabetics, 3(2.5\%) had clinical hypothyroidism, 5(4.1\%) had subclinical hypothyroidism and hyperthyroidism was noted in $1(0.8 \%)$ patient.

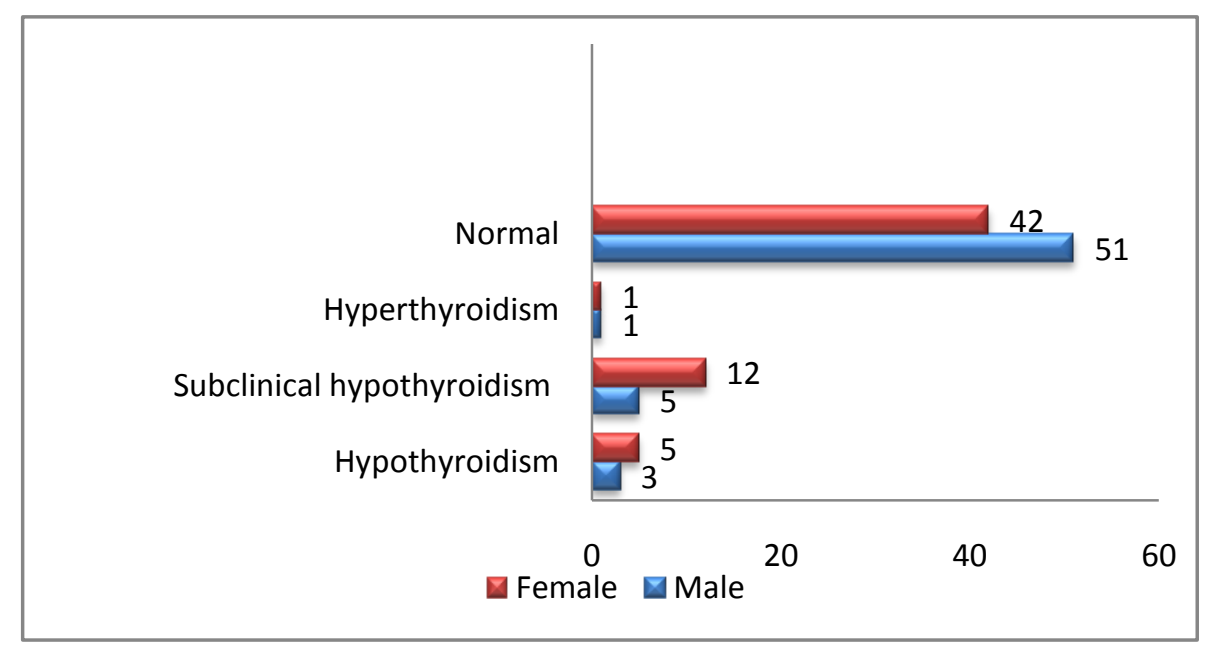

Table 3 shows age wise distribution of thyroid dysfunction in Type 2 Diabetes mellitus among male cases $(\mathrm{n}=60)$

\begin{tabular}{|l|l|l|l|l|}
\hline Age & Hypothyroidism & $\begin{array}{l}\text { Subclinical } \\
\text { hypothyroidism }\end{array}$ & Hyperthyroidism & Normal \\
\hline Below 60 years & 0 & 1 & 0 & 24 \\
\hline Above 60 years & 3 & 4 & 1 & 27 \\
\hline Total & 3 & 5 & 1 & 51 \\
\hline
\end{tabular}

In this study, 25 diabetics male were below 60 years and 35 diabetics were above 60 years. Among males, 8(6.6\%) patients of thyroid dysfunction was seen in above 60 years of age group, which comprises of 
hypothyroidism in 3 patients, subclinical hypothyroidism in 4 patients and hyperthyroidism in 1 patient . Only, $1(0.8 \%)$ patient of subclinical hypothyroidism was below 60 years of age.

Table 4 shows age wise distribution of thyroid dysfunction in Type 2 Diabetes mellitus among female cases $(\mathrm{n}=60)$

\begin{tabular}{|l|l|l|l|l|}
\hline Age & Hypothyroidism & $\begin{array}{l}\text { Subclinical } \\
\text { hypothyroidism }\end{array}$ & Hyperthyroidism & Normal \\
\hline Below 60 years & 1 & 5 & 0 & 22 \\
\hline Above 60 years & 4 & 7 & 1 & 20 \\
\hline Total & 5 & 12 & 1 & 42 \\
\hline
\end{tabular}

Among female patients, 32 patients were above 60 years. Thyroid dysfunction was seen in $12(10 \%)$ patients in above 60 years of age group, which comprises of clinical hypothyroidism in 4 patients, subclinical hypothyroidism in 7 patients and hyperthyroidism in 1 patient. In below 60 years of age group, out of 28 patients, only $6(5 \%)$ patients of thyroid dysfunction was noted. Subclinical hypothyroidism was seen in 5 patients.

Table 5: shows thyroid dysfunction in patient with diabetic complication.

\begin{tabular}{|l|l|l|}
\hline & Total case & Cases with thyroid dysfunction \\
\hline DM with microvascular complication. & 44 & 12 \\
\hline No complication & 76 & 15 \\
\hline Total & & \\
\hline
\end{tabular}

In the present study, microvascular complication was seen in 44 patients out of 120 patients. Among these 44 patients, 12 diabetics had thyroid dysfunction. Other 15 patients of thyroid dysfunction were seen in 76 cases of diabetes with no complication.

Table 6 shows thyroid dysfunction in patient with diabetic complications in details.

\begin{tabular}{|l|l|l|}
\hline DM with microvascular complication. & Total no. of pt. & Cases with thyroid dysfunction \\
\hline Retinopathy & 12 & 3 \\
\hline Neuropathy & 14 & 5 \\
\hline Nephropathy & 18 & 4 \\
\hline
\end{tabular}

In this study, we found out of 12 patients with retinopathy, 3 had thyroid disorders. Neuropathy was seen in 14 patients, out of which 5 had thyroid dysfunction. Nephropathy was diagnosed in 18 patients and thyroid dysfunction was present in 4 patients.

\section{Discussion}

This study comprises of 120 diabetic patients with equal number of male and female patients. All these patients hail from rural area of Assam. The study demonstrated a 22.5\% prevalence of thyroid dysfunction in diabetic patients. It was comparable to a Indian study done by Ravishankar et al,2013 (29\%) (1). Various workers reported a lower prevalence rate of thyroid dysfunction among diabetics from different countries in their study. A study conducted by Papazafiropoulou A et al (2010) found 12.3\% in Greek diabetics (2), Perros P et al (1995) reported $6.9 \%$ and another study by Radaideh A R M et al (2004) in Jordan found $12.5 \%$ of thyroid dysfunction in diabetic patients $(3,4)$. But ,one study carried out by Udiong C E J et al(2007) in calbar, Nigeria found a higher prevalence rate of thyroid dysfunction (46.5\%) (5).

In this study, among the thyroid spectrum disorders, subclinical hypothyroidism was the most frequent dysfunction, $14 \%$ of the patients. This finding was similar to studies done by various workers in different parts of the world.(1,2,4). Further, subclinical hypothyroidism was more frequent in females i.e $10 \%$ compared to males $4.1 \%$ which was comparable to a Indian study by Ravishankar et al in 2013 (1). Hyperthyroidism was equal in either sex with one patient in male and one patient in female. 
In our study, we have found $20(16.6 \%)$ patients with thyroid dysfunction over 60 years of age compared to 7 ( $5.8 \%$ ) patients below the age of 60 years. Many studies have shown that increased prevalence of hypothyroidism in elderly diabetics compared to young diabetics. Ravishankar et al reported $34.4 \%$ thyroid dysfunction over 60 years of age compared to that of $26.4 \%$ below the age of 60 years.

In the present study, microvascular complication was seen in 44 diabetic patients. Among the complications, retinopathy was found in 12 patients, neuropathy in 14 patients and nephropathy in 18 patients. Out of the 18 nephropathy cases, 4 had thyroid dysfunction. 5 patients had thyroid dysfunction among 14 diabetics with neuropathy. 3 patients of thyroid dysfunction was seen in 12 patients with retinopathy. This findings were comparable with the study conducted by Ravishankar et al in 2013 (1).

\section{Conclusion}

In conclusion, the present study showed a high prevalence of thyroid dysfunction $(22.5 \%)$ among the diabetic patients. Subclinical hypothyroidism was common than clinical hypothyroidism. Again, subclinical hypothyroidism was more frequent in females. Thyroid dysfunction was more common in elderly diabetics. Thyroid dysfunction aggravates dyslipidemia and hypertension in diabetic patients. Thus, undiagnosed thyroid dysfunction in diabetics increases the cardiovascular risk in these patients. Hence, screening for thyroid dysfunction among diabetic patients should be routinely performed to reduce the morbidity and mortality. However, more prospective studies with large sample size are necessary to know the impact of thyroid dysfunction in diabetes patients.

\section{Competeng interest: none}

\section{Reeference}

[1]. Ravishankar, S.N, Champakamalini, Venkatesh, Mohsin, A prospective study of thyroid dysfunction in patients with Type 2 diabetes in general population. Archives of Medicine 2013 vol.5 no. 1.2 doi : $10.3823 / 105$

[2]. Papazafiropoulou, A Prevalence of thyroid dysfunction among Greek Type2 diabetic patients attending an outpatient clinic. Journal of clinical Medicine Research 2010; 2(2): 75 - 78 .

[3]. Radaideh ,A. R. M, Nusier, M. K, Amari, F. L. et al. Thyroid dysfunction in patients with Type 2 diabetes mellitus in Jordan. Saudi Medical Journal 2004; $25(8): 1046$ - 1050.

[4]. Perros P, Mc Crimmon RJ, Shaw G, Frier BM. Frequency of thyroid dysfunction in diabetic patients: value of annual screening. Diabet Mwd . 1995; 12(7) : 622 - 627. Doi: 10 . 1111/j . 1464-5491. 1995. Tb00553.x.( pub med)

[5]. Udiong, C.E.J, Udoh ,A E , Etukudoh, ME. Evaluation of thyroid function in diabetes mellitus in Calabar, Nigeria. Indian Journal of Clinical Biochemistry 2007; 22(2) : 74- 78.

[6]. Chen G, Juan W, Yinghua L . et al. Associations between cardiovascular risk, insulin resistence, beta- cell function and thyroid dysfunction : a cross sectional study in S he ethnic minority group of Fujian Province in China. Euro J Endocrinology. 2010; 163:775- 782. Doi: 10. 1530/EJE - 10 -0710.(Pub Med). 